\title{
Antifúngicos em infecções oculares: drogas e vias de administração
}

\section{Antifungals in eye infections: drugs and routes of administration}

Guilherme Gubert Müller¹, Newton Kara-José2, Rosane Silvestre de Castro²

\section{ResUMO}

O tratamento das infecções oculares por fungos representa um desafio à prática oftalmológica. Para obtermos resposta terapêutica adequada, além do uso da droga correta, é necessária a administração desta de forma eficaz. Este manuscrito reúne informações a respeito das principais drogas antifúngicas utilizadas em infecções oculares, suas concentrações e principais vias de administração.

Descritores: Antimicóticos/uso terapêutico; Infecções oculares fúngicas; Micoses; Leveduras; Fungos filamentosos

\section{ABSTRACT}

Treatment of fungal eye infections represents a challenge to the ophthalmology practice. For an adequate therapeutic response, besides correct drug choice, it is necessary an effectively administration. This script gathers information about the major antifungal drugs used in eye infections, their concentrations and main administration routes.

Keywords: Antifungal agents/therapeutic use; Fungal eye infections; Mycoses; Yeasts; Filamentous fungi

\footnotetext{
'Programa de pós-graduação em Ciências Médicas em Oftalmologia, da Faculdade de Ciências Médicas da Universidade Estadual de Campinas (UNICAMP) - Campinas (SP), Brasil;

2Departamento de Oftalmologia da Faculdade de Ciências Médicas da Universidade Estadual de Campinas (UNICAMP) - Campinas (SP), Brasil.

Trabalho realizado Hospital das Clínicas da Universidade Estadual de Campinas (UNICAMP) - Campinas (SP), Brasil
}

O autor declara não haver conflitos de interesse

Recebido para publicação em: 28/11/2011 - Aceito para publicação em: 02/02/2012

Rev Bras Oftalmol. 2013; 72 (2): 132-41 


\section{INTRODUÇÃO}

A s infecções oculares por fungos são importantes causadoras de morbidade ocular. Desde o relato da primeira ceratite micótica por Leber em $1879^{(1}$, vimos um aumento crescente do número dos casos. Sugerem-se como principais fatores o uso de corticosteróides, facilitando a penetração do agente, e a popularização do uso de antibióticos tópicos, criando um ambiente de menor competitividade na superfície ocu$\operatorname{lar}^{(2,3)}$.

Apesar do surgimento de novos fármacos, a resolução permanece difícil em inúmeros casos. Comparados aos antibacterianos, os antifúngicos têm eficácia inferior, seja pelo mecanismo de ação dessas drogas (geralmente fungistáticos, com ação fungicida dose dependente), pela menor penetração tecidual ou, ainda, pelo caráter indolente da infecção ${ }^{(4)}$.

Este artigo tem por objetivo reunir informações a respeito dos principais antifúngicos utilizados atualmente pela oftalmologia no tratamento de ceratites e endoftalmites fúngicas, destacando suas vantagens e desvantagens a fim de facilitar a escolha da terapia mais adequada a cada caso.

\section{POLIENOS}

Pertencem a essa classe de antifúngicos a Anfotericina-B (AMB), a Nistatina e a Natamicina (NTM). A Nistatina deixou de ser utilizada em infecções oculares há algumas décadas devido à sua baixa penetração tecidual, toxicidade e relatos de resistência ${ }^{(5,6)}$. Entretanto, a AMB e a NTM mantêm-se como as principais drogas no manejo de infecções fúngicas oculares.

\section{- Anfotericina B}

Pertencente à família dos antibióticos macrolídeos poliênicos, a AMB foi o primeiro dos antifúngicos de amplo espectro a ser descoberto. Isolada na década de 50, é produto do actinomiceto Streptomyces nodosus. Seu uso tornou-se popular com a aprovação pelo FDA, na década de 60, e pela grande eficiência no controle de infecções fúngicas disseminadas ${ }^{(4,6-8)}$. Em oftalmologia, ainda é a droga de referência.

Seus mecanismos de ação são: aumento da permeabilidade celular, através da formação de poros ou canais ao ligar-se com o ergosterol da membrana celular fúngica, e ação oxidativa sobre as células, alterando suas funções metabólicas. Liga-se também ao colesterol das células humanas, principal razão de seus efeitos colaterais $^{(8,9)}$.

Seu nome deriva da sua propriedade anfotérica (solúvel em pH extremos, tanto ácidos como bases). Tem baixa hidrossolubilidade, sendo necessária a diluição em desoxicolato para administração. Possui moléculas longas que, ao serem infundidas, se aglutinam em um coloide. É foto e termossensível, devendo ser armazenada em local escuro e sob refrigeração (2 a $\left.8^{\circ} \mathrm{C}\right)^{(4,6,7,10)}$. Possui, invariavelmente, ação fungistática, com ação fungicida dependente da concentração atingida no tecido alvo ${ }^{(11)}$.

Em medicina interna tem uso restrito devido à sua toxicidade e efeitos colaterais. Durante a infusão podem ocorrer: febre, calafrios, taquipnéia, hipotensão, náuseas, vômitos entre outros. Sempre acarreta lesão tubular com perda da função renal em pacientes com doença renal prévia. Há também eliminação parcial via hepática ${ }^{(8,11,12)}$. Não deve ser diluída em solução salina, pois pode haver agregação dos coloides com consequente diminuição na disponibilidade da droga.

Possui atividade tanto contra fungos filamentares como leveduras. Apresenta excelente espectro contra Candida spp., Aspergillus spp., Penicilium marneffei, Criptococus spp. e agentes causadores da mucormicose. Eficaz também, porém em menor escala, contra as principais espécies de Fusarium. Não tem nenhuma ação antibacteriana ${ }^{(4)}$.

A AMB apresenta, também, ação imunopotenciadora através da ligação ao colesterol na membrana celular dos linfócitos. Os linfócitos T supressores apresentam maior concentração de colesterol em sua membrana do que os linfócitos B e T helper, o uso da AMB leva a uma consequente redução na população das células supressoras, com aumento relativo das células pré-inflamatórias ${ }^{(13,14)}$.

A administração sistêmica da AMB apresenta pouca penetração nos tecidos oculares, não atingindo níveis terapêuticos na córnea, aquoso ou vítreo ${ }^{(4,10,15-17)}$. Além disso, seus efeitos colaterais desestimulam o uso dessa via. A administração direta in situ acaba sendo a principal forma de tratamento. É uma das poucas drogas que apresenta descrição na literatura do uso subconjuntival, intraestromal, intracameral e intravítreo, além do seu uso tópico.

O uso tópico na concentração de 1,5 a $5 \mathrm{mg} / \mathrm{ml}$ é comumente a primeira escolha no tratamento das ceratomicoses. Deve ser preparada a partir da formulação endovenosa (Fungizone ${ }^{\circledR}$ Bristol-Meyers Squibb, New York,NY) diluída em água destilada. Seu uso é feito em intervalos de 1 em 1 hora no início do tratamento, com espaçamento para cada 4 horas após observado resposta terapêutica. É recomendado o debridamento periódico do epitélio corneano durante o tratamento, pois, o grande tamanho da molécula dificulta a penetração na córnea com epitélio íntegro. Em coelhos, a concentração de AMB após a administração tópica, com remoção do epitélio, conseguiu atingir níveis terapêuticos no estroma corneano. Já em córneas com o epitélio íntegro, a penetração foi baixa ou não foi possível detectar o fármaco ${ }^{(18-21)}$. O uso tanto na forma de colírios como pomada demonstram boa tolerabilidade e eficiência ${ }^{(22,23)}$.

A administração subconjuntival pode ser realizada em pacientes com baixa aderência ao tratamento, porém seu uso é limitado devido aos relatos de necrose conjuntival, esclerite e afinamento escleral ${ }^{(24,25)}$.

$\mathrm{O}$ uso intracorneano de $\mathrm{AMB}$, por outro lado, apresenta melhores resultados. Com poucos relatos de complicações, essa via de administração consegue atingir níveis terapêuticos corneanos superiores e por mais tempo que o uso tópico ou intracameral. Diversos casos de ceratites não responsivas ao tratamento tópico demonstram sucesso após a administração intraestromal ${ }^{(18,26)}$, porém, estudos controlados ainda são necessários. Sugere-se AMB via intraestromal na concentração de 5 a $10 \mu \mathrm{g}$ em infecções acometendo o estroma profundo que não respondam bem ao tratamento tópico ${ }^{(2)}$. O intervalo entre as aplicações deve ser de pelo menos sete dias e sob bloqueio peribulbar, devido à intensa dor causada pela aplicação. Doses acima de 15 a $20 \mu \mathrm{g}$ podem acarretar perda endotelial e edema corneano persistente ${ }^{(18)}$.

A injeção intracameral de AMB também pode ser realizada com concentração variando de 5 a $10 \mu \mathrm{g} / 0,1 \mathrm{ml}$. Administrada em intervalos de pelo menos um dia, pois há rápido escoamento da droga, não há perda endotelial significativa. É indicada em infecções profundas que penetram a membrana de Descemet e acometem a câmara anterior e/ou cristalino. Há relatos de catarata após as aplicações e aumento transitório da reação de câmara nas 24 horas subsequentes, devido ao caráter imunopotenciador da AMB. Outros efeitos colaterais, como irite e edema corneano, podem ocorrer, entretanto são reversíveis ${ }^{(27-31)}$.

Para o tratamento das endoftalmites fúngicas, a injeção intravítrea de AMB é a terapia de escolha. A dose recomendada varia de 1 a $10 \mu \mathrm{g} / 0,1 \mathrm{ml}$, podendo ser repetida semanalmen- 
te. Em pacientes vitrectomizados, o intervalo de administração deve ser reduzido para cada 3 ou 4 dias ${ }^{(32)}$. Estudos clínicos e experimentais demonstram segurança e eficácia com esta via de administração, porém, há relatos de toxicidade e necrose retiniana, provavelmente dose dependente ${ }^{(3,33)}$.

Nas infecções oculares por leveduras (em especial Candida spp.), a AMB ainda é a droga de primeira escolha. Embora o sucesso terapêutico dependa do uso da droga por tempo prolongado (pelo menos 4 semanas), poucos são os relatos de resistência a esses microrganismos ${ }^{(34)}$. Em infecções por fungos filamentosos, especialmente por Fusarium spp., há relatos de resistência ${ }^{(3,10)}$.

\section{- Natamicina}

Assim como a AMB, a NTM ou Pimaricina é um antifúngico polieno usado na medicina apenas no tratamento de ceratites fúngicas. Além disso, é utilizado como agrotóxico e na indústria alimentícia como conservante ${ }^{(3,35)}$.

Possui molécula longa com baixa solubilidade em água. Disponível em forma de suspensão, a NTM necessita ser agitada antes de sua aplicação. É a única droga aprovada pelo Food and Drug Administration - FDA para o tratamento de ceratomicoses. Alguns países possuem a droga em apresentação comercial (Natacyn ${ }^{\circledR}$ - Alcon Laboratories, Fort Worth, TX). No Brasil necessita ser manipulada ${ }^{(3,10)}$.

Utilizada na concentração de $5 \%(50 \mathrm{mg} / \mathrm{ml})$, apresenta boa estabilidade e tolerabilidade no uso tópico. Devido ao seu alto peso molecular, a NTM tem baixa penetração corneana e é indicada em esquema monoterápico apenas no tratamento de infecções superficiais ${ }^{(10,36)}$. Nos casos de infecções profundas ou com acometimento de estruturas intraoculares, o seu uso deve ser associado a antifúngico por outra via de administração ${ }^{(2,37-39)}$.

Devido à baixa penetração corneana, o sucesso terapêutico com NTM requer seu uso por tempo prolongado - média de 39 dias $^{(37)}$. Recomenda-se o debridamento do epitélio como terapia adjuvante para obter maiores concentrações estromais da NTM. Tal conduta permite maior aderência da droga à superfície desepitelizada ${ }^{(3,40)}$. Prajna et al. discordam em estudo que não revelou benefício da raspagem epitelial no tempo de cicatrização da lesão. De fato, em seu estudo, a raspagem esteve associada a pior acuidade visual após a cicatrização ${ }^{(41)}$.

O intervalo de aplicação é semelhante ao da AMB, havendo espaçamento das doses na melhora dos sintomas. Algumas infecções exigem a manutenção da terapia por períodos prolongados, o uso a cada 4 horas mantém concentrações terapêuticas na córnea com boa tolerabilidade em longo prazo ${ }^{(42)}$.

A aplicação subconjuntival deve ser desencorajada por sérias complicações, como necrose conjuntival e esclerite ${ }^{(3,24,43)}$. Não há relatos do uso da NTM por outras vias (intracameral, intravítrea, intraestromal ou sistêmica).

Possui amplo espectro de ação, especialmente contra fungos filamentares. Embora a NTM também possa ser utilizada em infecções por leveduras, a AMB mantém-se como droga de escolha devido a maior abrangência a diferentes espécies de Candida $^{(39,44)}$.

Infecções por Fusarium geralmente são tratadas com sucesso pela NTM, especialmente quando superficiais ${ }^{(45,46)}$. Lalitha et al., em estudo comparativo entre as concentrações inibitórias mínimas (MIC) dos antifúngicos, referiram que a NTM apresenta MIC relativo inferior ao da $\mathrm{AMB}$, tanto para as espécies isoladas de Fusarium quanto de Aspergillus ${ }^{(47)}$. Em outro estudo clínico, comparando a eficácia da NTM à do Voriconazol (VCZ), não houve diferença no tempo de cicatrização e acuidade visual final entre os dois grupos ${ }^{(41)}$. Kalavathy et al. ao comparar a eficácia da natamicina com a do fluconazol (FCZ) obtiveram, em- bora sem diferença significativa, melhores resultados no grupo tratado com NTM ${ }^{(45)}$. Diversos outros estudos também destacam a superioridade da NTM no tratamento de infecções por Fusarium $\operatorname{spp}^{(48-50)}$. Mesmo assim, alguns autores demonstram que cerca de 1/3 das infecções por Fusarium não responderam à $\mathrm{NTM}^{(37,51,52)}$. Nesses casos, a troca ou associação de outra droga é indicada.

\section{AZÓIS}

Introduzidos na prática médica na década de 70, os azóis representaram um importante avanço na terapia antifúngica. Comparados à AMB, possuem amplo espectro de ação e menores efeitos adversos. Seu uso se difundiu rapidamente, principalmente no tratamento de infecções de pele e mucosas ${ }^{(8)}$.

Atuam sobre enzimas do citocromo P450 dos fungos, bloqueando a síntese de ergosterol na membrana plasmática, inibindo, assim, o crescimento dos fungos. Divididos em duas grandes classes, os imidazóis foram os primeiros a surgir, seguidos pelos triazóis, ambos apresentam espectro antifúngico semelhante, com vantagem dos triazóis serem metabolizados mais lentamente e exercerem menor influência no metabolismo dos esteroides humanos ${ }^{(4,8)}$. Com metabolismo predominantemente hepático, sugere-se o controle durante o tratamento com essas drogas. Apresentam atividade teratogênica (classe C) e não devem ser usados durante a gestação ${ }^{(4,53)}$.

Entre os principais imidazóis utilizados em oftalmologia estão o miconazol (MCZ), o econazol (ECZ) e o cetoconazol $(\mathrm{KCZ})$. Dentre os triazóis de primeira geração, os mais utilizados são o itraconazol (ICZ) e o fluconazol. Introduzidos na prática clínica na última década estão os triazóis de segunda geração, entre eles o voriconazol e o posaconazol (PCZ).

\section{- Miconazol}

Desenvolvido para uso tópico como cremes para tratamento de pele e mucosas, o MCZ é utilizado principalmente no tratamento de micoses superficiais. Eficaz contra diversas cepas de Candida, é utilizado principalmente no tratamento de tinhas, candidíase vaginal e oral, devido à sua rápida ação fungicida sobre esses agentes ${ }^{(54)}$. Seu uso sistêmico, embora com bons resultados, está em desuso pelos efeitos colaterais cardiovasculares e hepatotóxicos ${ }^{(55,56)}$.

Além de atuar na síntese do ergosterol como os demais azóis, o MCZ promove inibição das peroxidades, resultando em acúmulo de radicais livres no citoplasma fúngico, levando à morte celular ${ }^{(57-60)}$.

O uso tópico, na concentração de $10 \mathrm{mg} / \mathrm{ml}$, apresenta boa penetração, especialmente se associado à remoção do epitélio ${ }^{(61-}$ ${ }^{63)}$. O uso de MCZ tópico como pomada também foi eficaz em estudo experimental, mantendo níveis terapêuticos mesmo com aplicações menos frequentes ${ }^{(64)}$. Seu uso ganhou destaque pela sua eficácia e segurança quando aplicado via subconjuntival (1,2 a 10mg) no tratamento de infecções por Candida, Fusarium, Curvalaria e Aspergillus ${ }^{(2,61,65,66)}$. O uso sistêmico não demonstrou concentração corneana e seu uso é desestimulado pelos seus efeitos adversos ${ }^{(61,67)}$.

Em comparação aos polienos, o MCZ apresenta menor eficácia terapêutica, embora apresente melhor penetração nos tecidos oculares ${ }^{(5,61)}$. In vitro, foi mais efetivo que o $\mathrm{KCZ}$ e o ICZ contra Aspergillus spp., Candida albicans e não-albicans ${ }^{(50,68)}$. Faltam estudos comparativos e controlados que comprovem o real benefício dessa droga.

\section{- Econazol}

Com estrutura molecular semelhante ao MCZ, o ECZ é um imidazólico utilizado inicialmente em micoses superficiais, com alguns estudos envolvendo seu uso sistêmico ${ }^{(69)}$.

Pouco estudado em infecções oculares, apresenta alguns 
relatos com seu uso tópico em ceratomicoses. Em um ensaio clínico controlado, comparando o ECZ colírio $2 \%(20 \mathrm{mg} / \mathrm{ml}) \mathrm{com}$ a NTM 5\%, não houve diferença estatística entre a taxa de sucesso terapêutico dos dois grupos, com bons resultados, sem relatos de reações adversas ${ }^{(42)}$. Mahashabde et al. sugerem o uso do ECZ 1\% pomada como tratamento profilático em traumas oculares com risco potencial de infecção fúngica ${ }^{(70)}$. Infelizmente, a ausência da droga disponível comercialmente para uso oftalmológico inviabiliza seu uso.

\section{- Cetoconazol}

Atualmente pouco utilizado em medicina interna, o $\mathrm{KCZ}$ foi o primeiro imidazol utilizado sistemicamente com sucesso. Sua substituição pelo ICZ deu-se pela menor influência deste no metabolismo dos glicocorticóides com espectro antifúngico ampliado $^{(4)}$. Utilizado na dose de 100 a 400mg cada 12 horas, tem sua absorção por via oral dependente do $\mathrm{pH}$ gástrico (inferior a 3 ), devendo ser tomado em jejum e não associado a protetores gástricos. Pode cursar com intolerância gástrica, hepatotoxicidade, ginecomastia e alterações menstruais ${ }^{(8,10)}$.

Embora sua penetração no líquor e na urina seja baixa, quando utilizado sistemicamente, a penetração no tecido ocular é significativa. Inúmeros são os relatos de sucesso do $\mathrm{KCZ}$ por via oral, associado ou não à NTM ou AMB tópicos, no tratamento de ceratomicoses. Alguns autores sugerem sua introdução na rotina de todos os casos de ceratites fúngicas ${ }^{(71-73)}$, porém faltam estudos controlados que o apoiem.

Há descrição de casos tratados exclusivamente com KCZ tópico $(10 \text { a } 50 \mathrm{mg} / \mathrm{ml})^{(74)}$, porém, outras drogas demonstraram superioridade em estudos comparativos. Komadina et al. e Singh et al. ao comparar o uso tópico e oral de KCZ à NTM demonstrou superioridade desta última droga. Obtiveram ainda resposta parcial ao uso oral isolado, com efeito aditivo quando associado à NTM tópica ${ }^{(75,76)}$.

Estudos in vitro com cepas de Aspergillus spp. e Fusarium spp. demonstraram menor suscetibilidade desses agentes ao $\mathrm{KCZ}$ quando comparados à NTM e VCZ ${ }^{(50)}$. Outros estudos laboratoriais também demonstram resultados semelhantes com cepas de Aspergillus, Fusarium e Candida spp. suscetíveis ao KCZ apenas em altas doses ${ }^{(34,77)}$.

Atualmente o uso sistêmico do $\mathrm{KCZ}$ é indicado no tratamento adjuvante de ceratomicoses profundas.

\section{- Itraconazol}

Mais comumente utilizado na prática geral que o $\mathrm{KCZ}$, o ICZ apresenta menores efeitos colaterais em seu uso sistêmico. Administrado por via oral, porém apresenta baixa biodisponibilidade, solubilidade e penetração nos tecidos oculares se comparado aos demais azóis ${ }^{(3,10,78,79)}$. Assim como o KCZ, sua absorção gástrica é dependente de baixo pH. Estudos em ratos demonstram menor risco teratogênico que o $\mathrm{KCZ}^{(53)}$.

Seu uso sistêmico na dose de $400 \mathrm{mg} /$ dia mostrou-se eficaz no tratamento de infecções por Candida spp. ${ }^{(80)}$. No entanto, em infecções por Fusarium spp. alguns estudos sugerem ineficácia do ICZ. Seu uso tópico na concentração de $10 \mathrm{mg} / \mathrm{ml}$ não foi efetivo como a NTM 5\% no controle da infecção ${ }^{(45)}$. Estudos in vitro demonstram MIC maior do ICZ em comparação à AMB e NTM ${ }^{(48,78)}$; alguns obtiveram inclusive resistência à droga por todas as cepas analisadas ${ }^{(47)}$. Contra Aspergillus spp. o ICZ mostrou-se eficaz, porém inferior ao $\mathrm{KCZ}^{(77)}$.

Seu uso sistêmico deve restringir-se apenas ao tratamento adjuvante de infecções oculares por leveduras.

\section{- Fluconazol}

Diferentemente do ICZ e do KCZ, o FCZ apresenta excelente absorção pelo trato gastrointestinal sem sofrer influência da acidez gástrica. Suas concentrações plasmáticas após o uso oral atingem praticamente os mesmos níveis que por via parenteral. Com eficaz penetração nos tecidos oculares, atinge concentrações no humor aquoso semelhante às do plasma ${ }^{(4,81)}$.

Seu uso por via oral na dose de 200 a $400 \mathrm{mg}$ por dia mostrou-se eficaz no tratamento de infecções oculares, associado ou não à NTM tópica ${ }^{(82,83)}$. Quando utilizado via subconjuntival, em associação à AMB tópica, houve aumento do espectro antifúngico com menor toxicidade que no uso isolado da $\mathrm{AMB}^{(84)}$. Yilmaz e Maden conseguiram tratar $60 \%$ dos casos de ceratomicoses com o uso isolado da injeção subconjuntival de $\mathrm{FCZ}^{(85)}$. Sugere-se o uso por essa via na dose de $2 \mathrm{mg}$ em $1 \mathrm{ml}$ administrado diariamente por 10 dias seguido por aplicações a cada 48 horas até melhora ${ }^{(86)}$.

Administrado como colírio em coelhos, o FCZ apresentou níveis terapêuticos intracorneanos contra cepas de Aspergillus fumigatus. Utilizado na concentração de $2 \mathrm{mg} / \mathrm{ml}$, sua penetração foi maior após remoção do epitélio ${ }^{(87,88)}$.

Seu uso no tratamento de endoftalmites fúngicas é preterido a outras drogas. Mesmo apresentando boa penetração vítrea quando administrada por via oral, sua ineficácia aos fungos filamentares desencorajam seu uso como adjuvante. Entretanto, há relatos de sucesso em casos de endoftalmite endógena por Candida $\operatorname{spp}^{(89-91)}$.

Embora com penetração ocular superior ao KCZ, estudos in vitro e in vivo demonstram menor espectro antifúngico do FCZ. Em diversos trabalhos que avaliaram a suscetibilidade de agentes isolados de ceratites ou endoftalmites fúngicas, apenas espécies de Candida foram sensíveis ao FCZ, com marcada resistência pelos fungos filamentares (Aspergillus e Fusarium spp.) $)^{(34,48,77,92)}$.

- Voriconazol

Mantendo o mesmo mecanismo de ação que os triazóis de primeira geração, o VCZ se difere desses por bloquear mais intensamente a síntese do ergosterol. Desenvolvido a partir da molécula do FCZ, apresenta maior eficácia em MIC's inferiores aos dos primeiros triazóis, o que aumenta sua ação contra fungos filamentares ${ }^{(8)}$. Devido à sua grande eficácia no tratamento de micoses disseminadas, com menor toxicidade em comparação à $\mathrm{AMB}$, o VCZ é, hoje em dia, a droga de escolha no tratamento da aspergilose invasiva ${ }^{(93)}$.

Disponível comercialmente nas formas oral e parenteral (Vfend ${ }^{\circledR}$ - Pfizer, New York, NY), o VCZ é metabolizado pelo fígado sendo necessário o controle de enzimas hepáticas durante o tratamento. Entre seus efeitos colaterais destacam-se os distúrbios visuais (visão borrada, alteração na percepção de cores e fotofobia) presentes em cerca de $30 \%$ dos usuários, normalmente reversíveis. Assim como o FCZ, apresenta boa biodisponibilidade e absorção gástrica ${ }^{(4,92)}$.

Administrado por via oral na dose de $200 \mathrm{mg}$ a cada 12 horas, atinge níveis máximos no plasma entre 2 e 3 horas. Tem sido amplamente estudado no tratamento de ceratites e endoftalmites pela capacidade de atingir boas concentrações nos diversos tecidos oculares (córnea, aquoso e vítreo) ${ }^{(32,94)}$. Hariprasad et al. observaram concentrações do VCZ no vítreo e aquoso de $38 \%$ e $51 \%$ dos níveis plasmáticos, respectivamente, após administração oral. Embora a concentração atingida no vítreo tenha sido insuficiente no tratamento de infecções por Fusarium spp., os autores defendem que o estudo foi realizado em olhos não inflamados e que, na vigência de inflamação, a quebra da barreira hemato-ocular auxiliaria no aumento da concentração local da droga ${ }^{(95)}$. Alfonso et al. sugere o VCZ como droga de primeira escolha para o uso via oral no tratamento de ceratites profundas, esclerites, endoftalmites e na profilaxia póstransplante penetrante de córnea ${ }^{(2)}$. Hariprasad et al. também 
sugere o VCZ oral como profilaxia nos casos de trauma ocular com material vegetal ${ }^{(92)}$.

Seu uso intravítreo demonstrou segurança em modelo experimental em ratos, não havendo alterações eletrorretinográficas com doses de até $25 \mathrm{mg} / \mathrm{ml}^{(96)}$.

Inúmeros também são os relatos de sucesso com o uso tópico de VCZ, administrado na concentração de $1 \mathrm{mg} / \mathrm{ml}$, foi eficaz no tratamento de ceratites por Candida, Aspergillus, Fusarium, Scedosporium, Paecilomyces entre outros ${ }^{(97-101)}$. Apresenta como vantagens aos polienos sua maior estabilidade a luz e temperatura, mantendo-se efetivo por até 30 dias $^{(102,103)}$. Estudos em cavalos demonstram a penetração da droga mesmo na vigência de integridade epitelial ${ }^{(104)}$.

Alguns relatos apoiam a injeção intracorneana de VCZ nos casos de ceratites profundas não responsivas ao tratamento tópico e/ou oral. Prakash et al. relatam sucesso em três casos de ceratite não responsiva à NTM tópica com o uso de VCZ $50 \mu \mathrm{g} /$ $0,1 \mathrm{ml}^{(105)}$. Recentemente Siatiri et al. descreveram 3 casos de ceratite por Fusarium não responsiva ao tratamento tópico que resolveram após a aplicação do VCZ intracorneano ${ }^{(106)}$, os autores sugerem que a aplicação direta do $\mathrm{VCZ}$ na córnea eleva sua concentração acima da concentração inibitória mínima dessas espécies de Fusarium. Sharma et al. em uma série de 13 pacientes também sugere o uso do VCZ intraestromal em ceratites refratárias ${ }^{(107)}$.

Entretanto, poucos são os estudos comparativos entre o $\mathrm{VCZ}$ e os demais antifúngicos. Em um estudo multicêntrico randomizado, não foi possível comprovar a superioridade do VCZ à NTM, com ambos os grupos apresentando tempo de cicatrização e acuidade visual final semelhantes ${ }^{(41)}$. Há Inclusive relatos

Tabela 1

Antifúngicos e suas indicações

\begin{tabular}{|c|c|c|c|}
\hline Droga & $\begin{array}{l}\text { Via } \\
\text { administração }\end{array}$ & Dose & Indicação \\
\hline \multirow[t]{4}{*}{ Anfotericina B } & Tópico & 1,5 a $5 \mathrm{mg} / \mathrm{ml}$ & $\begin{array}{l}\text { - Primeira escolha no tratamento de ceratites por leveduras } \\
\text { - Alternativa à NTM no tratamento de ceratites por fungos } \\
\text { filamentares }\end{array}$ \\
\hline & Intraestromal & 5 a $10 \mu g$ & - Ceratites profundas com resposta parcial à droga tópica \\
\hline & Intracameral & 5 a $10 \mu \mathrm{g} / 0,1 \mathrm{ml}$ & - Ceratites com acometimento de câmara anterior e/ou cristalino \\
\hline & Intravítrea & 1 a $10 \mu \mathrm{g} / 0,1 \mathrm{ml}$ & $\begin{array}{l}\text { - Primeira escolha no tratamento de endoftalmites fúngicas (leveduras } \\
\text { ou fungos filamentares) }\end{array}$ \\
\hline Natamicina & Tópica & $50 \mathrm{mg} / \mathrm{ml}$ & $\begin{array}{l}\text { - Primeira escolha no tratamento de ceratomicoses por fungos } \\
\text { filamentares }\end{array}$ \\
\hline Miconazol & Subconjuntival & 1,2 a $10 \mathrm{mg} / 1 \mathrm{ml}$ & $\begin{array}{l}\text { - Alternativa à AMB no tratamento de ceratites por leveduras } \\
\text { - Adjunto à terapia tópica em pacientes com baixa aderência } \\
\text { ao tratamento }\end{array}$ \\
\hline Econazol & Tópico & $20 \mathrm{mg} / \mathrm{ml}$ & - Alternativa à NTM em ceratites por fungos filamentares \\
\hline Cetoconazol & Oral & $\begin{array}{l}100 \text { a } 400 \mathrm{mg} \\
\text { de } 12 / 12 \mathrm{hs}\end{array}$ & $\begin{array}{l}\text { - Adjunto à terapia tópica em ceratites profundas ou } \\
\text { com acometimento intraocular }\end{array}$ \\
\hline Itraconazol & Oral & $400 \mathrm{mg} / \mathrm{dia}$ & $\begin{array}{l}\text { - Adjunto à terapia tópica em ceratites profundas } \\
\text { ou com acometimento intraocular por leveduras }\end{array}$ \\
\hline \multirow[t]{3}{*}{ Fluconazol } & Tópico & $2 \mathrm{mg} / \mathrm{ml}$ & - Alternativa aos polienos do tratamento de ceratomicoses \\
\hline & Subconjuntival & $2 \mathrm{mg} / 1 \mathrm{ml}$ & - Adjunto em pacientes com baixa adesão ao tratamento \\
\hline & Oral & 200 a 400mg/dia & $\begin{array}{l}\text { - Adjunto à terapia tópica em ceratites profundas ou com } \\
\text { acometimento intraocular }\end{array}$ \\
\hline \multirow[t]{6}{*}{ Voriconazol } & Tópico & $1 \mathrm{mg} / \mathrm{ml}$ & - Ceratomicoses resistente aos polienos e triazóis de primeira linha \\
\hline & Intraestron & $50 \mu \mathrm{g} / 0,1 \mathrm{ml}$ & $\begin{array}{l}\text { - Ceratites profundas com resposta parcial à droga tópica ou } \\
\text { baixa adesão ao tratamento }\end{array}$ \\
\hline & Intracameral & $50 \mu \mathrm{g} / 0,1 \mathrm{ml}$ & - Ceratomicoses com acometimento de câmara anterior e/ou cristalino \\
\hline & Intravítrea & $50 \mu \mathrm{g} / 0,1 \mathrm{ml}$ & - Alternativa à AMB nas endoftalmites fúngicas \\
\hline & Oral & $200 \mathrm{mg}$ de $12 / 12 \mathrm{hs}$ & $\begin{array}{l}\text { - Adjunto à terapia tópica em ceratites profundas } \\
\text { ou com acometimento intraocular }\end{array}$ \\
\hline & & & - Profilaxia pós-trauma com material vegetal \\
\hline \multirow[t]{2}{*}{ Posaconazol } & Tópico & $100 \mathrm{mg} / \mathrm{ml}$ & - Ceratomicoses resistente aos polienos e triazóis de primeira linha \\
\hline & Oral & $\begin{array}{l}200 \mathrm{mg} \text { de } 6 / 6 \mathrm{hs} \text { ou } \\
400 \mathrm{mg} \text { de } 12 / 12 \mathrm{hs}\end{array}$ & $\begin{array}{l}\text { - Adjunto em ceratites profundas e endoftalmite } \\
\text { por fungos resistentes aos polienos e triazóis de primeira linha }\end{array}$ \\
\hline Flucitocina & Tópica & $10 \mathrm{mg} / \mathrm{ml}$ & - Em ceratomicoses associado à AMB tópica por leveduras \\
\hline Caspofungina & Tópica & 1,5 a $5 \mathrm{mg} / \mathrm{ml}$ & $\begin{array}{l}\text { - Ceratomicoses por levedura resistente aos polienos e } \\
\text { triazóis de primeira linha }\end{array}$ \\
\hline Micafungina & Tópica & $1 \mathrm{mg} / \mathrm{ml}$ & $\begin{array}{l}\text { - Ceratomicoses por levedura resistente aos polienos e } \\
\text { triazóis de primeira linha }\end{array}$ \\
\hline
\end{tabular}


Figura 1. Esquema terapêutico para Ceratítes Fúngicas

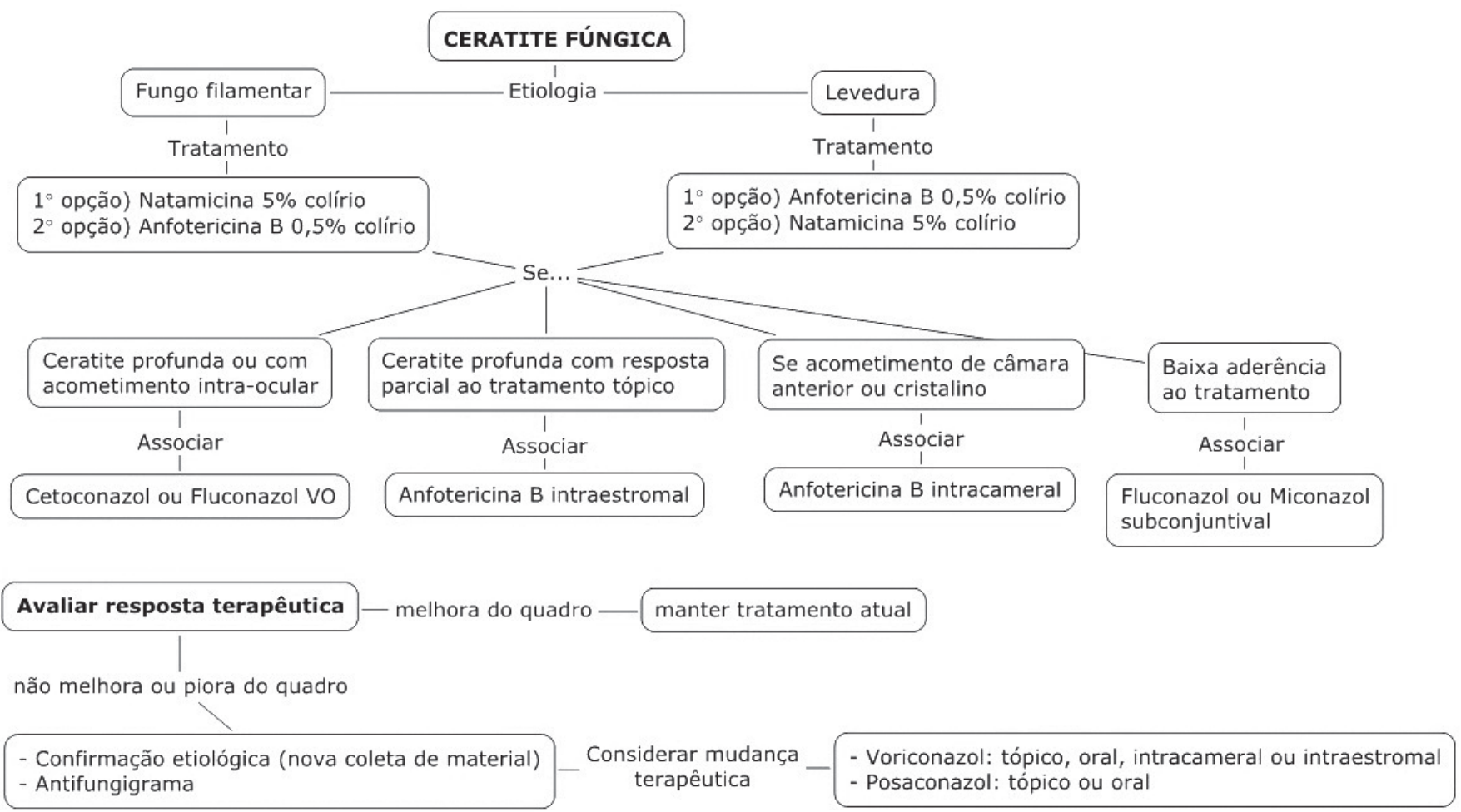

de falha terapêutica com o uso do VCZ. Giaconi et al. reportam dois casos, uma ceratite por Fusarium oxysporum e outra por Colletotrichum dematium que não responderam a terapia tópica com VCZ ${ }^{(108)}$

Estudos in vitro demonstram superioridade do VCZ sobre a AMB contra Aspergillus spp ${ }^{(109-112)}$. Contra espécies de Fusarium, o MIC absoluto do VCZ, NTM e AMB foram semelhantes, com o MIC relativo do VCZ inferior ao dos polienos ${ }^{(47)}$. Mesmo assim, a concentração inibitória mínima às espécies de Fusarium foi superior quando comparada às de espécies de Candida e Aspergillus ${ }^{(77)}$.

\section{- Posaconazol}

Assim como o VCZ, o PCZ é um triazol de segunda geração recentemente introduzido na prática médica. Resultado do aperfeiçoamento da molécula do ICZ, sua principal indicação está no tratamento de infecções fúngicas invasivas em pacientes onco-hematológicos. Disponível apenas em solução oral (Noxafil ${ }^{\circledR}$ - Schering-Plough, Kenilworth, NJ), deve ser administrado na dose de $200 \mathrm{mg}$, 4 vezes ao dia, ou $400 \mathrm{mg}$, duas vezes ao dia. A apresentação parenteral encontra-se em desenvolvimento. Queixas gastrointestinais foram os únicos efeitos adversos relatados até o momento ${ }^{(113)}$.

Estudos in vitro e in vivo demonstram amplo espectro contra Candida spp., Criptococcus neoformans, Aspergillus spp., Fusarium spp. entre outros. Foi eficaz contra a maioria dos agentes resistentes ao ICZ e FCZ ${ }^{(114,115)}$, apresentando, juntamente com o VCZ, as menores MIC's contra diversos agentes ${ }^{(47)}$.

Sua experiência em infecções oculares ainda é restrita, entretanto com resultados animadores. Em uma série de três casos de ceratite por Fusarium, evoluindo com endoftalmite não responsiva ao tratamento oral e tópico com $\mathrm{VCZ}$, houve rápida resposta terapêutica ao $\mathrm{PCZ}^{(36)}$. Sponsel et al. também descrevem um caso de ceratite por Fusarium solani resistente à AMB e NTM, tratado com sucesso após introdução de PCZ 200mg, 4 vezes ao dia, via oral, associado ao uso tópico $(100 \mathrm{mg} / \mathrm{ml}$ preparado a partir da solução oral) ${ }^{(116)}$. Porém, faltam estudos controlados e comparativos com drogas antifúngicas de primeira linha.

\section{PIRIMIDINAS}

Representada pela 5-fluorcitocina (5FC) ou flucitocina, é a única droga antifúngica com ação intracelular. Quando absorvida pelo fungo é convertida em 5-fluoracila, potente antimetabólico, que atua inibindo a síntese do $\mathrm{DNA}^{(4,117)}$.

Seu uso em infecções oculares é restrito devido ao seu estreito espectro antifúngico e baixa penetração nos tecidos oculares ${ }^{(17)}$. É eficaz contra Candida spp. e possui ação variada contra Aspergillus spp. Não possui ação sobre Fusarium spp. Seu uso sistêmico ou tópico deve ser feito juntamente com AMB, primeiramente pelo seu efeito potencializador (sinergismo) e pela indução de resistência ao uso isolado do $5 \mathrm{FC}^{(4,6,77,118)}$.

\section{EQUINOCANDINAS}

Equinocandinas são lipopeptídeos semissintéticos que inibem a síntese de glucana na parede celular do fungo através da inibição não competitiva da enzima 1,3- $\beta$-glucano sintase, causando desequilíbrio osmótico e lise celular ${ }^{(8,119,120)}$. São representantes dessa classe de medicamentos a caspofungina (CFG) e a micafungina (MFG).

Utilizado em infecções por leveduras, tem rápida ação fungicida contra a maioria das espécies de Candida, inclusive cepas resistentes ao FCZ, mas não contra Cryptococcus, Rhodotorula e Trichosporon ${ }^{(121)}$. Fungistática contra alguns fun- 
gos filamentares como Aspergillus, mas sem atuação sobre Fusarium e Rhizopus ${ }^{(47,122)}$. A CFG é administrada por via endovenosa (Cancidas ${ }^{\circledR}$ - Merck \& Co - Whitehouse Station, NJ) na dose de $70 \mathrm{mg}$ no primeiro dia, com redução para 50mg nos dias seguintes $^{(4,8)}$. E a MFG (Mycamine ${ }^{\circledR}$ - Astellas Ireland Killorglin,Irlanda) é administrada na dose de 100 a 150mg/dia, também por via parenteral.

Utilizada topicamente na concentração 1,5 a $5 \mathrm{mg} / \mathrm{ml}$, a CFG mostrou eficácia semelhante à AMB no tratamento de úlcera corneana por Candida albicans em modelo animal ${ }^{(123)}$. Outros dois estudos envolvendo o uso tópico do MFG $1 \mathrm{mg} / \mathrm{ml}$ mostraram eficácia comparável ou superior a do FCZ no tratamento de ceratites por Candida albicans e Candida parapsilosis ${ }^{(124,125)}$.

\section{ASSOCIAÇÃO DE ANTIFÚNGICOS}

No intuito de aumentar a eficiência no tratamento ou até mesmo ampliar o espectro antifúngico, é comum associarmos drogas no tratamento de infecções oculares. Embora algumas combinações de antifúngicos, tal como $5 \mathrm{FC}$ e $\mathrm{AMB}$ tenham uso consagrado $^{(126)}$, outras associações menos estudadas podem não atender as nossas expectativas.

É comum observamos a associação de um azol à terapia antifúngica tópica padrão com NTM ou AMB. Entretanto diversos estudos demonstram ação antagônica entre essas drogas. Ao introduzirmos um azol, diminuímos a síntese do ergosterol da membrana celular, sítio de ligação dos polienos, com consequente perda da função deste.

Arora et al. observaram esse efeito antagônico ao associar o uso de ECZ e AMB no tratamento de ceratomicoses, obtendo o mesmo resultado terapêutico com o uso isolado do $\mathrm{ECZ}^{(127)}$. Em estudo de revisão, Sugar et al. demonstram efeito antagônico in vitro entre AMB e diversos azóis (MCZ, KCZ, FCZ e ICZ), com perda da ação do polieno ${ }^{(128)}$. Li et al., em estudo semelhante, observaram antagonismo na combinação NTM + ICZ e NTM + FCZ, e sinergismo entre AMB e ICZ ${ }^{(48)}$.

Estudos em humanos e animais comumente não reproduzem esses achados laboratoriais. Inúmeros são os relatos de melhora na associação de antifúngicos, principalmente na combinação AMB tópica com triazóis de primeira e segunda geração sistêmicos ${ }^{(129,130)}$. Tal combinação deve ser utilizada em infecções corneanas profundas ou com acometimento intraocular.

A associação de duas drogas de mesma classe deve ser desencorajada (ex. NTM + AMB), pois, além de aumentar a toxicidade local, não há aumento na eficácia terapêutica ${ }^{(131)}$.

\section{OUTRAS DROGAS}

$\mathrm{O}$ uso de terapêuticas alternativas aos antifúngicos tem sido estudado com o intuito de tratar ceratites com diagnóstico suspeito ou interrogado. Em uma série de casos, o uso de iodo povidona $2,3 \%$ foi utilizado no tratamento de ceratites por Candida albicans e Acremonium strictuim com bons resulta$\operatorname{dos}^{(132)}$. Porém, num estudo comparativo, a iodo povidona $0,5 \%$ não demonstrou benefício quando comparado a NTM $5 \%$ no tratamento de ceratites experimentais por Fusarium solani ${ }^{(40)}$. Em outro estudo experimental, Fiscella et al. demonstraram eficácia no tratamento com Polihexametileno biguanida (PHBM) a 0,02\% em olhos de coelhos infectados por Fusarium solani ${ }^{(133)}$. Porém, não há registros de estudos comparativos entre PHMB e antifúngicos.

Ensaios experimentais associando corticosteróides tópicos à terapia antifúngica mostram efeito deletério ao tratamento. O'Day et al. demonstraram modificação da resposta do hospedeiro após introdução do corticoide. Em seu estudo, coelhos infectados por Candida albicans, Aspergillus fumigatus e
Fusarium solani, que receberam corticóide subconjuntival, apresentaram retardo na esterilização corneana em comparação ao grupo controle ${ }^{(134)}$. Weiyun et al. estudando fatores de risco para recorrência da infecção fúngica após transplante, referem aumento de seis vezes no risco de recorrência da infecção em pacientes que receberam corticoide tópico prévio ao transplante ${ }^{(135)}$.

\section{Conclusão}

Pudemos observar que várias são as opções de drogas e vias de administração dos antifúngicos, e que sua escolha depende tanto do agente etiológico quanto da localização e extensão da infecção (Tabela 1).

A terapia padrão, baseada nos polienos, ainda se mantém eficaz. Embora existam inúmeros relatos de infecções que não respondem às drogas de primeira linha, com melhora após a introdução de novas medicações, em especial os triazóis de segunda geração, mas faltam estudos comparativos que demonstrem a superioridade desses últimos.

Até que se prove o real benefício da nova geração de antifúngicos, entendemos que tais medicamentos devem ser utilizados como alternativa à terapia padrão (Figura 1).

\section{REFERÊNCIAS}

1. LeberTH. Keratomycosis aspergillina als ursache von hypopyonkeratites. Graefes Ach Clin Exp Ophthalmol. 1879;25:285-301.

2. Alfonso EC GA, Miller D. Fungal keratitis. In: Krachmer JH, Mannis MJ,Holland EJ, editors. Cornea: fundamentals, diagnosis and management. 3rd ed. New York: Mosby Elsevier; 2011.

3. Jones D. Diagnosis and management of fungal keratitis. In:Tasman W, Jaeger EA, editors. Duane's clinical ophthalmology. Philadelphia: Lippincott Williams \& Wilkins; 2004.

4. Bennett J.Agentes antimicrobianos, agentes antifúngicos. In: Brunton L, editor. Goodman \& Gilman: as bases farmacológicas da terapêutica. 11a ed. Rio de Janeiro: McGraw-Hill; c2007. p. 1103-17.

5. Reddy PR, Reddy PS, Reddy AR, Saboo NK. A comparative evaluation of Nystatin, Amphotericin-B and Miconazole in keratomycosis. Indian J Ophthalmol. 1982;30(4):249-50.

6. Oliveira PR, Resende SM, Oliveira FC, Oliveira AC. Ceratite fúngica. Arq Bras Oftalmol. 2001;64(1):75-9.

7. Filippin FB, Souza LC.Eficiência terapêutica das formulações lipídicas de anfotericina B. RBCF Rev Bras Ciênc Farm. 2006;42(2):167-94.

8. Martinez R. Atualização no uso de agentes antifúngicos. J Bras Pneumol. 2006;32(5):449-60.

9. Gallis HA, Drew RH, Pickard WW. Amphotericin B: 30 years of clinical experience. Rev Infect Dis. 1990;12(2):308-29.

10. Kaur IP, Rana C, Singh H. Development of effective ocular preparations of antifungal agents. J Ocul Pharmacol Ther. 2008;24(5):481-93. Review.

11. Khoo SH, Bond J, Denning DW. Administering amphotericin B-a practical approach. J Antimicrob Chemother. 1994;33(2):203-13.

12. Mora-Duarte J,Betts R, Rotstein C, Colombo AL, Thompson-Moya L, Smietana J, Lupinacci R, Sable C, Kartsonis N, Perfect J; Caspofungin Invasive Candidiasis Study Group. Comparison of caspofungin and amphotericin B for invasive candidiasis. N Engl J Med. 2002;347(25):2020-9. Comment in N Engl J Med. 2002;347(25):20702. ACP J Club. 2003;139(1):15. N Engl J Med. 2003;348(13):1287-8; author reply 1287-8.

13. Shirley SF, Little JR. Immunopotentiating effects of amphotericin B. I. Enhanced contact sensitivity in mice. J Immunol. 1979;123(6):2878-82.

14. Shirley SF, Little JR. Immunopotentiating effects of amphotericin B. II. Enhanced in vitro proliferative responses of murine lymphocytes. J Immunol. 1979;123(6):2883-9. 
15. Green WR, Bennett JE, Goos RD. Ocular penetration of amphoteri$\operatorname{cin}$ B: a report of laboratory studies and a case report of postsurgical cephalosporium endophthalmitis. Arch Ophthalmol. 1965;73:769-75.

16. Goldblum D, Frueh BE, Zimmerli S, Böhnke M. Treatment of postkeratitis fusarium endophthalmitis with amphotericin B lipid complex. Cornea. 2000;19(6):853-6.

17. O'Day DM, Head WS, Robinson RD, Stern WH, Freeman JM. Intraocular penetration of systemically administered antifungal agents. Curr Eye Res. 1985;4(2):131-4. Erratum in: Curr Eye Res 1986;5(7):547.

18. Qu L, Li L, Xie H. Corneal and aqueous humor concentrations of amphotericin B using three different routes of administration in a rabbit model. Ophthalmic Res. 2010;43(3):153-8.

19. Pleyer U, Grammer J, Pleyer JH, Kosmidis P, Friess D, Schmidt KH, Thiel HJ. [Amphotericin B-bioavailability in the cornea. Studies with local administration of liposome incorporated amphotericin B]. Ophthalmologe. 1995;92(4):469-75. German.

20. O'Day DM, Head WS, Robinson RD, Clanton JA. Corneal penetration of topical amphotericin B and natamycin. Curr Eye Res. 1986;5(11):877-82.

21. O'Day DM, Ray WA, Head WS, Robinson RD. Influence of the corneal epithelium on the efficacy of topical antifungal agents. Invest Ophthalmol Vis Sci. 1984;25(7):855-9.

22. Hirose H, Terasaki H, Awaya S, Yasuma T. Treatment of fungal corneal ulcers with amphotericin B ointment. Am J Ophthalmol. 1997;124(6):836-8.

23. Wood TO,Williford W.Treatmen of keratomycosis with amphotericin B 0.15\%. Am J Ophthalmol. 1976;81(6):847-9.

24. O'Day DM. Selection of appropriate antifungal therapy. Cornea. 1987;6(4):238-45. Review.

25. O'Day DM, Ray WA, Robinson RD, Head WS, Williams TE. Differences in response in vivo to amphotericin B among Candida albicans strains. Invest Ophthalmol Vis Sci. 1991;32(5):1569-72.

26. Garcia-Valenzuela E, Song CD. Intracorneal injection of amphothericin B for recurrent fungal keratitis and endophthalmitis. Arch Ophthalmol. 2005;123(12):1721-3.

27. Kermani NK, Aggarwal SP. Isolated post-operative Aspergillus niger endophthalmitis. Eye (Lond). 2000;14(Pt 1):114-6.

28. Yoon KC, Jeong IY, Im SK, Chae HJ, Yang SY. Therapeutic effect of intracameral amphotericin B injection in the treatment of fungal keratitis. Cornea. 2007;26(7):814-8.

29. Kaushik S, Ram J, Brar GS, Jain AK, Chakraborti A, Gupta A. Intracameral amphotericin B: initial experience in severe keratomycosis. Cornea. 2001;20(7):715-9.

30. Kuriakose T, Kothari M, Paul P, Jacob P, Thomas R. Intracameral amphotericin B injection in the management of deep keratomycosis. Cornea. 2002;21(7):653-6.

31. Yilmaz S, Ture M, Maden A. Efficacy of intracameral amphotericin B injection in the management of refractory keratomycosis and endophthalmitis. Cornea. 2007;26(4):398-402.

32. Perraut LE Jr, Perraut LE, Bleiman B, Lyons J. Successful treatment of Candida albicans endophthalmitis with intravitreal amphotericin B. Arch Ophthalmol. 1981;99(9):1565-7.

33. Serracarbassa PD, Peyman GA, Liang C, Calixto N Jr, Nair MG. Toxicity and efficacy of intravitreal injection of spartanamicin B in the treatment of Candida endophthalmitis. Int Ophthalmol. 1998;22(1):53-8.

34. Mascaro VLDM, Höfling-Lima AL, Gompertz OF, Yu MCZ, Matta DA, Colombo AL. Antifungal susceptibility testing of yeast isolated from corneal infections. Arq Bras Oftalmol. 2003;66(5):647-52.

35. Mattia A, Cerniglia C, Baines J. Safety evaluation of certain food additives and contaminants. Natamycin (Pimaricin). WHO Food Additives Series: 48. Available from: http://www.inchem.org/documents/jecfa/ jecmono/v48je06.htm.

36. Tu EY, McCartney DL, Beatty RF, Springer KL, Levy J, Edward D. Successful treatment of resistant ocular fusariosis with posaconazole (SCH-56592). Am J Ophthalmol. 2007;143(2):222-7.

37. Jones DB, Sexton R, Rebell G. Mycotic keratitis in South Florida: a review of thirty-nine cases. Trans Ophthalmol Soc U K. 1970;89:781-97.
38. Panda A, Sharma N,Das G, Kumar N, Satpathy G. Mycotic keratitis in children: epidemiologic and microbiologic evaluation. Cornea. 1997;16(3):295-9.

39. Rosa RH Jr, Miller D, Alfonso EC. The changing spectrum of fungal keratitis in south Florida. Ophthalmology. 1994;101(6):1005-13.

40. Oliveira LA, Takata TT, Shiguematsu AI, Melo Júnior LA, Gompertz OF, Sousa LB, Mannis MJ. Effect of topical $0.5 \%$ povidone-iodine compared to $5 \%$ natamycin in fungal keratitis caused by Fusarium solani in a rabbit model: a pilot study. Arq Bras Oftalmol. 2008;71(6):860-4.

41. Prajna NV, Mascarenhas J,Krishnan T, Reddy PR, Prajna L, Srinivasan $\mathrm{M}$, et al. Comparison of natamycin and voriconazole for the treatment of fungal keratitis. Arch Ophthalmol. 2010;128(6):672-8. Comment in Arch Ophthalmol. 2011;129(6):814; author reply 814-5.

42. Prajna NV, John RK, Nirmalan PK, Lalitha P, Srinivasan M. A randomised clinical trial comparing $2 \%$ econazole and $5 \%$ natamycin for the treatment of fungal keratitis. $\mathrm{Br} \mathrm{J}$ Ophthalmol. 2003;87(10):1235-7.

43. Dursun D, Fernandez V, Miller D, Alfonso EC. Advanced fusarium keratitis progressing to endophthalmitis. Cornea. 2003;22(4):300-3.

44. Tanure MA, Cohen EJ, Sudesh S, Rapuano CJ, Laibson PR. Spectrum of fungal keratitis at Wills Eye Hospital, Philadelphia, Pennsylvania. Cornea. 2000;19(3):307-12.

45. Kalavathy CM, Parmar P, Kaliamurthy J,Philip VR, Ramalingam MD Jesudasan CA,Thomas PA. Comparison of topical itraconazole 1\% with topical natamycin $5 \%$ for the treatment of filamentous fungal keratitis. Cornea. 2005;24(4):449-52.

46. Klont RR, Eggink CA, Rijs AJ, Wesseling P, Verweij PE. Successful treatment of Fusarium keratitis with cornea transplantation and topical and systemic voriconazole. Clin Infect Dis. 2005;40(12):e110-2.

47. Lalitha P, Shapiro BL, Srinivasan M, Prajna NV,Acharya NR, Fothergill AW, et al. Antimicrobial susceptibility of Fusarium, Aspergillus, and other filamentous fungi isolated from keratitis. Arch Ophthalmol. 2007;125(6):789-93.

48. Li L, Wang Z, Li R, Luo S, Sun X. In vitro evaluation of combination antifungal activity against Fusarium species isolated from ocular tissues of keratomycosis patients. Am J Ophthalmol. 2008;146(5):724-8.

49. Xuguang S, Zhixin W, Zhiqun W, Shiyun L, Ran L. Ocular fungal isolates and antifungal susceptibility in northern China. Am J Ophthalmol. 2007;143(1):131-3

50. Pearce JW, Giuliano EA, Moore CP. In vitro susceptibility patterns of Aspergillus and Fusarium species isolated from equine ulcerative keratomycosis cases in the midwestern and southern United States with inclusion of the new antifungal agent voriconazole. Vet Ophthalmol. 2009;12(5):318-24.

51. Jones DB, Forster FK, Rebell G. Fusarium solani keratitis treated with natamycin (pimaricin): eighteen consecutive cases. Arch Ophthalmol. 1972;88(2):147-54

52. Polack FM, Kaufman HE, Newmark E. Keratomycosis. Medical and surgical treatment. Arch Ophthalmol. 1971;85(4):410-6.

53. Amaral VCS, Nunes Junior GP. Ketoconazole- and fluconazole-induced embryotoxicity and skeletal anomalies in wistar rats: a comparative study. Braz Arch Biol Technol. 2008;51(6):1153-61.

54. Sawyer PR, Brogden RN, Pinder RM, Speight TM, Avery GS Miconazole: a review of its antifungal activity and therapeutic efficacy. Drugs. 1975;9(6):406-23.

55. Coley KC, Crain JL. Miconazole-induced fatal dysrhythmia. Pharmacotherapy. 1997;17(2):379-82.

56. Barasch A, Griffin AV. Miconazole revisited: new evidence of antifungal efficacy from laboratory and clinical trials. Future Microbiol. 2008;3(3):265-9.

57. Fothergill AW. Miconazole: a historical perspective. Expert Rev Anti Infect Ther. 2006;4(2):171-5.

58. Kobayashi D, Kondo K, Uehara N, Otokozawa S, Tsuji N, Yagihashi A, Watanabe N. Endogenous reactive oxygen species is an important mediator of miconazole antifungal effect. Antimicrob Agents Chemother. 2002;46(10):3113-7. 
59. Thevissen K, Ayscough KR, Aerts AM, Du W, De Brucker K, Meert EM, et al. Miconazole induces changes in actin cytoskeleton prior to reactive oxygen species induction in yeast. J Biol Chem. 2007;282(30):21592-7.

60. Sud IJ, Feingold DS. Mechanisms of action of the antimycotic imidazoles. J Invest Dermatol. 1981;76(6):438-41.

61. Foster CS, Stefanyszyn M. Intraocular penetration of miconazole in rabbits. Arch Ophthalmol. 1979;97(9):1703-6.

62. Mohan M, Panda A, Gupta SK. Management of human keratomycosis with miconazole. Aust N Z J Ophthalmol. 1989;17(3):295-7.

63. Fitzsimons R, Peters AL. Miconazole and ketoconazole as a satisfactory first-line treatment for keratomycosis. Am J Ophthalmol. 1986;101(5):605-8

64. Gupta SK. Efficacy of miconazole in experimental keratomycosis. Aust N Z J Ophthalmol. 1986;14(4):373-6.

65. Foster CS. Miconazole therapy for keratomycosis. Am J Ophthalmol. 1981;91(5):622-9.

66. Ishibashi Y, Kaufman HE. The effects of subconjunctival miconazole in the treatment of experimental Candida keratitis in rabbits. Arch Ophthalmol. 1985;103(10):1570-3.

67. Thomas PA. Current perspectives on ophthalmic mycoses. Clin Microbiol Rev. 2003;16(4):730-97.

68. Uchida K, Abe S, Yamaguchi H. The postantifungal effect (PAFE) of itraconazole, in comparison with those of miconazole and fluconazole, on Candida Species. Microbiol Immunol. 2006;50(9):679-85.

69. Heel RC,Brogden RN,Speight TM,Avery GS. Econazole: a review of its antifungal activity and therapeutic efficacy. Drugs. 1978;16(3):177-201.

70. Mahashabde S, Nahata MC, Shrivastava U. A comparative study of anti-fungal drugs in mycotic corneal ulcer. Indian J Ophthalmol. 1987;35(5-6):149-52.

71. Ishibashi Y. Oral ketoconazole therapy for keratomycosis. Am J Ophthalmol. 1983;95(3):342-5.

72. Thomas PA,Kalavathy CM,Abraham DJ,Rajasekaran J.Oral ketoconazole in Keratomycosis. Indian J Ophthalmol. 1987;35(4):197-203.

73. Ishibashi Y. Miconazole and ketoconazole as a satisfactory first-line treatment for keratomycosis. Am J Ophthalmol. 1986;102(4):547-8.

74. Torres MA, Mohamed J, Cavazos-Adame H, Martinez LA. Topical ketoconazole for fungal keratitis. Am J Ophthalmol. 1985;100(2):293-8.

75. Komadina TG, Wilkes TD, Shock JP, Ulmer WC, Jackson J, Bradsher RW. Treatment of Aspergillus fumigatus keratitis in rabbits with oral and topical ketoconazole. Am J Ophthalmol. 1985;99(4):476-9.

76. Singh SM, Khan R, Sharma S, Chatterjee PK. Clinical and experimental mycotic corneal ulcer caused by Aspergillus fumigatus and the effect of oral ketoconazole in the treatment. Mycopathologia. 1989;106(3):133-41.

77. Marangon FB, Miller D, Giaconi JA, Alfonso EC. In vitro investigation of voriconazole susceptibility for keratitis and endophthalmitis fungal pathogens. Am J Ophthalmol. 2004;137(5):820-5.

78. Rajasekaran J, Thomas PA, Kalavathy CM, Joseph PC, Abraham DJ Itraconazole therapy for fungal keratitis. Indian J Ophthalmol. 1987;35(5-6):157-60

79. Abad JC,Foster CS. Fungal keratitis. Int Ophthalmol Clin.1996;36(3):115. Review

80. Klotz SA,Zahid M, Bartholomew WR, Revera PM, Butrus S. Candida albicans keratitis treated successfully with itraconazole. Cornea. 1996;15(1):102-4.

81. O'Day DM, Foulds G, Williams TE, Robinson RD, Allen RH, Head WS. Ocular uptake of fluconazole following oral administration. Arch Ophthalmol. 1990;108(7):1006-8.

82. Thakar M. Oral fluconazole therapy for keratomycosis. Acta Ophthalmol (Copenh). 1994;72(6):765-7.

83. Urbak SF, Degn T. Fluconazole in the management of fungal ocular infections. Ophthalmologica. 1994;208(3):147-56.

84. Mahdy RA, Nada WM, Wageh MM, Kader MA, Saleh MM, Alswad MM.Assessment safety and efficacy of a combination therapy of topical amphotericin B and subconjunctival fluconazole for the treatment of fungal keratitis. Cutan Ocul Toxicol. 2010;29(3):193-7.
85. Yilmaz S, Maden A. Severe fungal keratitis treated with subconjunctival fluconazole. Am J Ophthalmol. 2005;140(3):454-8. Comment in Am J Ophthalmol. 2006;141(4):783; author reply 783-4.

86. Mahdy RA, Nada WM, Wageh MM. Topical amphotericin B and subconjunctival injection of fluconazole (combination therapy) versus topical amphotericin B (monotherapy) in treatment of keratomycosis. J Ocul Pharmacol Ther. 2010;26(3):281-5.

87. Yee RW, Cheng CJ, Meenakshi S, Ludden TM, Wallace JE, Rinaldi MG. Ocular penetration and pharmacokinetics of topical fluconazole. Cornea. 1997;16(1):64-71. Comment in Cornea. 1997;16(6):700.

88. Avunduk AM, Beuerman RW, Warnel ED, Kaufman HE, Greer D. Comparison of efficacy of topical and oral fluconazole treatment in experimental Aspergillus keratitis. Curr Eye Res. 2003;26(2):113-7.

89. Dotto PF, Rodrigues LD, Fernandes MLB, Figueira BBD, Takahashi WY, Carani JC, Serracarbassa PD. Uso do fluconazol endovenoso no tratamento da endoftalmite fúngica endógena: relato de caso. Arq Bras Oftalmol. 2005;68(4):543-6.

90. Christmas NJ, Smiddy WE. Vitrectomy and systemic fluconazole for treatment of endogenous fungal endophthalmitis. Ophthalmic Surg Lasers. 1996;27(12):1012-8.

91. Wellington M, Gigliotti F. Update on antifungal agents. Pediatr Infect Dis J. 2001;20(10):993-5.

92. Hariprasad SM, Mieler WF, Lin TK, Sponsel WE, Graybill JR Voriconazole in the treatment of fungal eye infections: a review of current literature. Br J Ophthalmol. 2008;92(7):871-8.

93. Herbrecht R, Denning DW, Patterson TF, Bennett JE, Greene RE Oestmann JW, Kern WV, Marr KA, Ribaud P, Lortholary O, Sylvester R, Rubin RH, Wingard JR, Stark P, Durand C, Caillot D, Thiel E, Chandrasekar PH, Hodges MR, Schlamm HT, Troke PF, de Pauw B; Invasive Fungal Infections Group of the European Organisation for Research and Treatment of Cancer and the Global Aspergillus Study Group. Voriconazole versus amphotericin B for primary therapy of invasive aspergillosis. N Engl J Med. 2002;347(6):408-15. Comment in N Engl J Med. 2002;347(25):2080-1; author reply 2080-1. N Engl J Med. 2002;347(25):2080-1; author reply 2080-1. N Engl J Med. 2004;350(9):950-2.

94. Freda R. Use of oral voriconazole as adjunctive treatment of severe cornea fungal infection: case report. Arq Bras Oftalmol. 2006;69(3):431-4.

95. Hariprasad SM, Mieler WF, Holz ER, Gao H, Kim JE, Chi J, Prince RA. Determination of vitreous, aqueous, and plasma concentration of orally administered voriconazole in humans. Arch Ophthalmol. 2004;122(1):42-7.

96. Gao H, Pennesi M, Shah K, Oiao X, Hariprasad SM, Mieler WF, et al Safety of intravitreal voriconazole: electroretinographic and histopathologic studies. Trans Am Ophthalmol Soc. 2003;101:183-9; discussion 189.

97. Anderson KL, Mitra S, Salouti R, Pham TA, Taylor HR. Fungal keratitis caused by Paecilomyces lilacinus associated with a retained intracorneal hair. Cornea. 2004;23(5):516-21.

98. Bunya VY,Hammersmith KM, Rapuano CJ,Ayres BD, Cohen EJ.Topical and oral voriconazole in the treatment of fungal keratitis. Am J Ophthalmol. 2007;143(1):151-3.

99. Lee SJ, Lee JJ, Kim SD. Topical and oral voriconazole in the treatment of fungal keratitis. Korean J Ophthalmol. 2009;23(1):46-8.

100. Nulens E, Eggink C, Rijs AJ, Wesseling P,Verweij PE. Keratitis caused by Scedosporium apiospermum successfully treated with a cornea transplant and voriconazole. J Clin Microbiol. 2003;41(5):2261-4

101. Polizzi A, Siniscalchi C, Mastromarino A, Saccà SC. Effect of voriconazole on a corneal abscess caused by fusarium. Acta Ophthalmol Scand. 2004;82(6):762-4.

102. Al-Badriyeh D, Neoh CF, Stewart K, Kong DC. Clinical utility of voriconazole eye drops in ophthalmic fungal keratitis. Clin Ophthalmol. 2010;4:391-405.

103. Dupuis A, Tournier N, Le Moal G,Venisse N. Preparation and stability of voriconazole eye drop solution. Antimicrob Agents Chemother. 2009;53(2):798-9. 
104. Clode AB, Davis JL, Salmon J, Michau TM, Gilger BC. Evaluation of concentration of voriconazole in aqueous humor after topical and oral administration in horses. Am J Vet Res. 2006;67(2):296-301.

105. Prakash G, Sharma N, Goel M, Titiyal JS, Vajpayee RB. Evaluation of intrastromal injection of voriconazole as a therapeutic adjunctive for the management of deep recalcitrant fungal keratitis. Am J Ophthalmol. 2008;146(1):56-9.

106. Siatiri H, Daneshgar F, Siatiri N, Khodabande A. The effects of intrastromal voriconazole injection and topical voriconazole in the treatment of recalcitrant Fusarium keratitis. Cornea. 2011;30(8):872-5.

107. Sharma N, Agarwal P, Sinha R, Titiyal JS, Velpandian T, Vajpayee RB. Evaluation of intrastromal voriconazole injection in recalcitrant deep fungal keratitis: case series. Br J Ophthalmol. 2011;95(12):1735-7.

108. Giaconi JA, Marangon FB, Miller D, Alfonso EC. Voriconazole and fungal keratitis: a report of two treatment failures. J Ocul Pharmacol Ther. 2006;22(6):437-9.

109. Diekema DJ, Messer SA, Hollis RJ, Jones RN, Pfaller MA. Activities of caspofungin, itraconazole, posaconazole, ravuconazole, voriconazole, and amphotericin B against 448 recent clinical isolates of filamentous fungi. J Clin Microbiol. 2003;41(8):3623-6.

110. Johnson LB, Kauffman CA. Voriconazole: a new triazole antifungal agent. Clin Infect Dis. 2003;36(5):630-7.

111. Espinel-Ingroff A, Boyle K, Sheehan DJ. In vitro antifungal activities of voriconazole and reference agents as determined by NCCLS methods: review of the literature. Mycopathologia. 2001;150(3):101-15.

112. Marco F, Pfaller MA, Messer SA, Jones RN. Antifungal activity of a new triazole, voriconazole (UK-109,496), compared with three other antifungal agents tested against clinical isolates of filamentous fungi. Med Mycol. 1998;36(6):433-6.

113. Ullmann AJ, Cornely OA, Burchardt A, Hachem R, Kontoyiannis DP, Töpelt K, et al. Pharmacokinetics, safety, and efficacy of posaconazole in patients with persistent febrile neutropenia or refractory invasive fungal infection. Antimicrob Agents Chemother. 2006;50(2):658-66.

114. Cuenca-Estrella M, Gomez-LopezA, Mellado E, Buitrago MJ,Monzon A, Rodriguez-Tudela JL. Head-to-head comparison of the activities of currently available antifungal agents against 3,378 Spanish clinical isolates of yeasts and filamentous fungi. Antimicrob Agents Chemother. 2006;50(3):917-21.

115. Torres HA, Hachem RY, Chemaly RF, Kontoyiannis DP, Raad II. Posaconazole: a broad-spectrum triazole antifungal. Lancet Infect Dis. 2005;5(12):775-85.

116. Sponsel WE, Graybill JR, Nevarez HL, Dang D. Ocular and systemic posaconazole(SCH-56592) treatment of invasive Fusarium solani keratitis and endophthalmitis. Br J Ophthalmol. 2002;86(7):829-30.

117. Vermes A, Guchelaar HJ, Dankert J. Flucytosine: a review of its pharmacology, clinical indications, pharmacokinetics, toxicity and drug interactions. J Antimicrob Chemother. 2000;46(2):171-9.

118. Mabon M. Fungal keratitis. Int Ophthalmol Clin. 1998;38(4):115-23.

119. Morris MI, Villmann M. Echinocandins in the management of invasive fungal infections, part 1. Am J Health Syst Pharm. 2006;63(18):1693-703. Review.

120. Morris MI, Villmann M. Echinocandins in the management of invasive fungal infections, Part 2. Am J Health Syst Pharm. 2006;63(19):1813-20. Review.

121. Bachmann SP, VandeWalle K, Ramage G, Patterson TF, Wickes BL, Graybill JR, López-Ribot JL. In vitro activity of caspofungin against Candida albicans biofilms. Antimicrob Agents Chemother. 2002;46(11):3591-6.
122. Wagner C, Graninger W, Presterl E, Joukhadar C. The echinocandins: comparison of their pharmacokinetics, pharmacodynamics and clinical applications. Pharmacology. 2006;78(4):161-77. Review.

123. Goldblum D, Frueh BE, Sarra GM, Katsoulis K, Zimmerli S. Topical caspofungin for treatment of keratitis caused by Candida albicans in a rabbit model. Antimicrob Agents Chemother. 2005;49(4):1359-63.

124. Matsumoto Y, Dogru M, Goto E, Fujishima H, Tsubota K. Successful topical application of a new antifungal agent, micafungin, in the treatment of refractory fungal corneal ulcers: report of three cases and literature review. Cornea. 2005;24(6):748-53.

125. Matsumoto Y, Murat D, Kojima T, Shimazaki J, Tsubota K. The comparison of solitary topical micafungin or fluconazole application in the treatment of Candida fungal keratitis. Br J Ophthalmol. 2011;95(10):1406-9.

126. Bennett JE, Dismukes WE, Duma RJ, Medoff G, Sande MA, Gallis H, et al. A comparison of amphotericin B alone and combined with flucytosine in the treatment of cryptoccal meningitis. N Engl J Med. 1979;301(3):126-31.

127. Arora I, Kulshrestha OP, Upadhaya S. Treatment of fungal corneal ulcers with econazole. Indian J Ophthalmol. 1983;31 Suppl:1019-21.

128. Sugar AM. Use of amphotericin B with azole antifungal drugs: what are we doing? Antimicrob Agents Chemother. 1995;39(9):1907-12.

129. Cacciapuoti A, Gurnani M, Halpern J, Norris C, Patel R, Loebenberg D. Interaction between posaconazole and amphotericin B in concomitant treatment against Candida albicans in vivo. Antimicrob Agents Chemother. 2005;49(2):638-42.

130. Najvar LK, Cacciapuoti A, Hernandez S, Halpern J, Bocanegra R, Gurnani M, et al. Activity of posaconazole combined with amphotericin B against Aspergillus flavus infection in mice: comparative studies in two laboratories. Antimicrob Agents Chemother. 2004;48(3):75864. Comment in Antimicrob Agents Chemother. 2004;48(8):3211; author reply 3211-2.

131. Lin HC, Chu PH, Kuo YH, Shen SC. Clinical experience in managing Fusarium solani keratitis. Int J Clin Pract. 2005;59(5):549-54.

132. Ndoye Roth PA, Ba EA, Wane AM, De Meideros M, Dieng M, Ka A, et al. [Fungal keratitis in an intertropical area: diagnosis and treatment problems. Advantage of local use of polyvidone iodine]. J Fr Ophtalmol. 2006;29(8):e19. French.

133. Fiscella RG, Moshifar M, Messick CR, Pendland SL, Chandler JW, Viana M. Polyhexamethylene biguanide (PHMB) in the treatment of experimental Fusarium keratomycosis. Cornea. 1997;16(4):447-9.

134. O'Day DM, Ray WA, Head WS, Robinson RD, Williams TE. Influence of corticosteroid on experimentally induced keratomycosis. Arch Ophthalmol. 1991;109(11):1601-4.

135. Shi W, Wang T, Xie L, Li S, Gao H, Liu J, Li H. Risk factors, clinical features, and outcomes of recurrent fungal keratitis after corneal transplantation. Ophthalmology. 2010;117(5):890-6.

\section{Autor Correspondente:}

Guilherme Gubert Müller

Av. Des. Hugo Simas, no 333

CEP 80520-250 - Curitiba (PR), Brasil

e-mail: guimuller@ig.com.br 